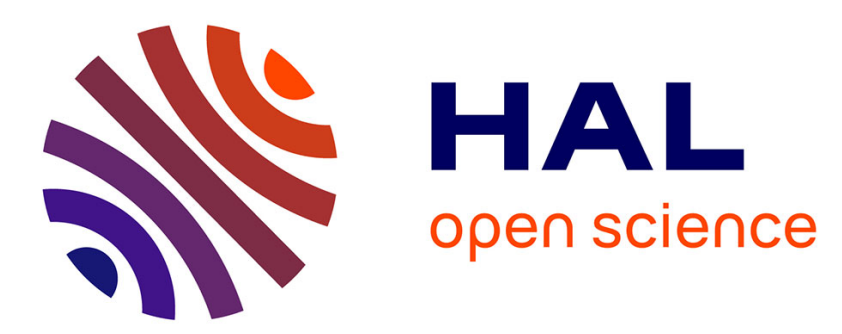

\title{
Stability of nonlinear delay systems : delay-independent small gain theorem and frequency domain interpretation of the Lyapunov-Krasovskii method
}

Pierre-Alexandre Bliman

\section{- To cite this version:}

Pierre-Alexandre Bliman. Stability of nonlinear delay systems : delay-independent small gain theorem and frequency domain interpretation of the Lyapunov-Krasovskii method. [Research Report] RR-3969, INRIA. 2000. inria-00072679

\section{HAL Id: inria-00072679 \\ https://hal.inria.fr/inria-00072679}

Submitted on 24 May 2006

HAL is a multi-disciplinary open access archive for the deposit and dissemination of scientific research documents, whether they are published or not. The documents may come from teaching and research institutions in France or abroad, or from public or private research centers.
L'archive ouverte pluridisciplinaire HAL, est destinée au dépôt et à la diffusion de documents scientifiques de niveau recherche, publiés ou non, émanant des établissements d'enseignement et de recherche français ou étrangers, des laboratoires publics ou privés. 


\section{Stability of nonlinear delay systems: delay-independent small gain theorem and frequency domain interpretation of the Lyapunov-Krasovskii method}

Pierre-Alexandre Bliman

\section{$\mathbf{N}^{\circ} 3969$}

Juillet 2000

THÈME 4 



\title{
Stability of nonlinear delay systems: delay-independent small gain theorem and frequency domain interpretation of the Lyapunov-Krasovskii method
}

\author{
Pierre-Alexandre Bliman* \\ Thème 4 - Simulation et optimisation \\ de systèmes complexes \\ Projet Sosso \\ Rapport de recherche $\mathrm{n}^{\circ} 3969$ - Juillet $2000-15$ pages
}

\begin{abstract}
The purpose of this note is to study the relationship between a certain stability criterion for nonlinear delay systems, obtained via Lyapunov-Krasovskii method, and a delay-independent version of the small gain theorem. We show that, contrary to the delay-free case (in which KalmanYakubovich-Popov lemma ensures the equivalence of the two approaches), the first method assumes stronger hypothesis than the second one. However, numerical verification of the former is in general NP-hard, whereas the latter leads to linear matrix inequalities. The difference between the two approaches is precisely stated, and, among other benefits, this permits to exhibit classes of problems for which the Lyapunov-Krasovskii method is not conservative.
\end{abstract}

Key-words: delay systems, stability, small gain theorem, quadratic Lyapunov-Krasovskii functionals, frequency domain.

* Email: pierre-alexandre.bliman@inria.fr 


\section{Stabilité des systèmes non-linéaires à retard: théorème des petits gains indépendant du retard et interprétation fréquentielle de la méthode de Lyapunov-Krasovskii}

Résumé : Le dessein de cette note est l'étude de la relation existant entre un certain critère de stabilité pour les systèmes non-linéaires à retard, obtenu par la méthode de Lyapunov-Krasovskii, et une version indépendante du retard du théorème des petits gains. Nous montrons que, contrairement à ce qui se passe pour le cas sans retard (pour lequel le lemme de Kalman-Yakubovich-Popov assure l'équivalence des deux approches), la première méthode est plus restrictive que la seconde. Cependant, la vérification numérique de celle-ci est en général un problème NP-difficile, alors que celle-là conduit à des inégalités linéaires matricielles. La différence entre les deux approches est clairement formulée et, entre autres bénéfices, ceci permet d'exhiber des classes de problèmes pour lesquels la méthode de Lyapunov-Krasovskii n'est pas conservative.

Mots-clés : systèmes à retard, stabilité, théorème des petits gains, fonctionnelles de LyapunovKrasovskii quadratiques, domaine fréquentiel. 


\section{Introduction}

The use of quadratic Lyapunov-Krasovskii functionals permits to obtain stability criteria for nonlinear delay systems, with hypotheses which do not depend upon the value of the delay [14, 20]. The goal of the present paper is to establish a frequency domain interpretation of this result and to examine the conservativity inherent to this approach. It turns out that this criterion is related to a "delayindependent small gain theorem", obtained from the usual small gain theorem by decoupling the terms in $s$ and $z=e^{-h s}$ in the transfer function of the system. This is reminiscent of some recent results on delay-independent stability of linear delay systems $[10,11,12,7,6]$.

For rational systems, the fact that one may equivalently obtain small gain theorem by frequency domain approach or by Lyapunov method with quadratic Lyapunov functions is derived from KalmanYakubovich-Popov lemma. The main result presented here (Theorem 1) establishes a similar relationship for delay systems. More precisely, it is shown that the success of the Lyapunov-Krasovskii functional approach, that is the solvability of the involved linear matrix inequality (LMI (17) below), is sufficient, but not necessary, to have small gain theorem for any value of the delay. Moreover, the use of a robust control approach already applied to linear delay systems $[4,8]$ and which amounts to interpret the delay and the nonlinearity as uncertainties, permits to express with precision the difference between the two types of conditions. It also shows that the numerical verification of the delay-independent small gain condition is in general a NP-hard problem. On the contrary, the criterion obtained via Lyapunov-Krasovskii approach may be easily checked numerically, providing possibly conservative evaluations.

In the remaining of the introduction are given some more details on the background (Sections 1.1 to 1.3). The main result (Theorem 1) is stated and commented in Section 2. Some applications of the latter are given in Section 3. In Section 3.1 are presented some classes of systems for which the Lyapunov-Krasovskii approach is not conservative. In Section 3.2, it is shown how this approach permits a natural extension of the theory to systems with commensurate delays; the same idea is exploited in [1], to get LMI stability criterion for systems with multiple independent delays, and to derive a LMI condition related to "delay-independent Popov criterion". A proof of Theorem 1 is given in Section 4.

\subsection{Small gain theorem for rational systems: equivalence between frequency do- main and Lyapunov approaches}

Consider the negative feedback interconnection of a memoryless multivariable time-varying nonlinearity $\psi$ with a linear time-invariant system characterized by its transfer function matrix $H(s)$ of size $p \times q$. Nonlinearity $\psi: \mathbb{R}^{+} \times \mathbb{R}^{p} \rightarrow \mathbb{R}^{q}$ is assumed to fulfill a growth condition: there exists $K \in \mathbb{R}^{q \times p}$ such that

$$
\forall t \geq 0, \forall y \in \mathbb{R}^{p}, \quad\|\psi(t, y)\| \leq\|K y\|,
$$

where $\|\cdot\|$ denotes the euclidian norm. The absolute stability problem consists in seeking conditions on $H$ and $K$ for stability of the interconnection, for any $\psi$ fulfilling (1).

When $H$ represents a strictly proper rational system $(A, B, C)$, that is

$$
H(s)=C(s I-A)^{-1} B,
$$

the system may be realized as:

$$
\dot{x}=A x-B \psi(t, y), \quad y=C x,
$$

where $A \in \mathbb{R}^{n \times n}, B \in \mathbb{R}^{n \times q}, C \in \mathbb{R}^{p \times n}$. Searching for a Lyapunov function of the type $V(x)=x^{T} P x$ for a certain positive definite matrix $P \in \mathbb{R}^{n \times n}$, one verifies that the inequality

$$
\frac{d}{d t}[V(x(t))] \leq\left(\begin{array}{c}
x(t) \\
\psi(t, y(t))
\end{array}\right)^{T}\left(\begin{array}{cc}
A^{T} P+P A+C^{T} K^{T} K C & -P B \\
-B^{T} P & -I
\end{array}\right)\left(\begin{array}{c}
x(t) \\
\psi(t, y(t))
\end{array}\right)
$$

$\mathrm{RR} \mathrm{n}^{\circ} 3969$ 
is fulfilled along the trajectories of (2), so the assumption that

$$
\exists P=P^{T}>0, \quad R=\left(\begin{array}{cc}
A^{T} P+P A+C^{T} K^{T} K C & -P B \\
-B^{T} P & -I
\end{array}\right)<0
$$

is sufficient for absolute stability. Problem (3) is a linear matrix inequality (LMI), for which there exist powerful numerical methods of resolution [2].

Kalman-Yakubovich-Popov lemma permits to express equivalently the previous condition under a frequency domain form, namely

$$
\forall s \in \mathbb{C} \text { with } \operatorname{Re} s \geq 0, \operatorname{det}(s I-A) \neq 0 \text { and }\|K H(s)\|<1,
$$

where $\|\cdot\|$ denotes here the largest singular value. This absolute stability criterion, the small gain theorem, may also be proved directly under the form (4), by use of input-output techniques [18, 22].

\subsection{Two absolute stability criteria for nonlinear delay systems}

Let us now consider the following nonlinear delay system (where $A, A_{d} \in \mathbb{R}^{n \times n}, B \in \mathbb{R}^{n \times q}, C, C_{d} \in$ $\left.\mathbb{R}^{p \times n}\right)$ :

$$
\dot{x}=A x+A_{d} x(t-h)-B \psi(t, y), y=C x+C_{d} x(t-h),
$$

where the nonlinearity $\psi$ is supposed to fulfill (1). System (5) realizes the interconnection of $\psi$ with the nonrational transfer

$$
H(s)=\left(C+e^{-s h} C_{d}\right)\left(s I-A-e^{-s h} A_{d}\right)^{-1} B
$$

For such a system, small gain theorem still holds [18, 22]: condition (4) (replacing $\operatorname{det}(s I-A)$ by $\operatorname{det}\left(s I-A-e^{-s h} A_{d}\right)$, and with $H$ now given by (6)) is sufficient for absolute stability of system (5) under hypothesis (1). This frequency domain condition depends explicitly upon the magnitude of the delay $h$.

On the other hand, it is possible to obtain absolute stability criterion by use of a LyapunovKrasovskii functional $V: \mathcal{C}([-h, 0]) \rightarrow \mathbb{R}$ of the type [14]

$$
V(\phi) \stackrel{\text { def }}{=} \phi^{T}(0) P \phi(0)+\int_{-h}^{0} \phi^{T}(s) Q \phi(s) d s
$$

where $P, Q$ are positive definite matrices. Similarly to the rational case, one proves that

$$
\frac{d}{d t}\left[V\left(x_{t}\right)\right] \leq\left(\begin{array}{c}
x(t) \\
x(t-h) \\
\psi(t, y(t))
\end{array}\right)^{T} R\left(\begin{array}{c}
x(t) \\
x(t-h) \\
\psi(t, y(t))
\end{array}\right)
$$

along the trajectories of (5), for a certain matrix $R$ affine in $P$ and $Q$ (see formula (17) below). In the previous inequality, $x_{t}$ denotes as usual the mapping $x(\cdot+t)$, defined on $[-h, 0]$. To get asymptotic stability, one is then led to assume the existence of positive definite $P$ and $Q$ such that $R<0$. The delay does not appear in this LMI: contrary to small gain theorem, the obtained result is a delay-independent criterion. Until now no relationship has been established between these two different approaches, which however both appear as natural extensions of the methods used for delay-free systems. 


\subsection{Delay-independent stability of linear delay systems}

Important progresses have been made recently in the study of delay-independent stability conditions for linear delay systems, see $[13,16]$. By definition, the linear equation

$$
\dot{x}=A x+A_{d} x(t-h)
$$

is asymptotically stable independently of the delay $[11,12]$ if

$$
\forall h \geq 0, \forall s \in \mathbb{C} \text { with } \operatorname{Re} s \geq 0, \operatorname{det}\left(s I-A-e^{-h s} A_{d}\right) \neq 0 .
$$

To analyze the conditions under which this property occurs, one sees that it is necessary to disconnect the term $e^{-s h}$ from $s$. Hale et al. [6] have shown that delay-independent (asymptotic) stability of the previous linear system is equivalent to ( $\sigma$ denotes the spectrum):

$$
\operatorname{Re} \sigma\left(A+A_{d}\right)<0 \text { and } \forall(s, z) \in j \mathbb{R} \times \mathbb{C} \text { with } s \neq 0,|z|=1, \operatorname{det}\left(s I-A-z A_{d}\right) \neq 0,
$$

a condition equivalent [7] to

$$
\operatorname{det}\left(A+A_{d}\right) \neq 0 \text { and } \forall(s, z) \in \mathbb{C}^{2} \text { with } \operatorname{Re} s \geq 0, s \neq 0,|z| \leq 1, \operatorname{det}\left(s I-A-z A_{d}\right) \neq 0 .
$$

Niculescu et al. [15, 16] have given numerical methods of verification of this condition. They also provide method to check a slightly stronger one, called strong delay-independent stability, namely

$$
\operatorname{Re} \sigma\left(A+A_{d}\right)<0 \text { and } \forall(s, z) \in j \mathbb{R} \times \mathbb{C} \text { with }|z|=1, \operatorname{det}\left(s I-A-z A_{d}\right) \neq 0 .
$$

The latter property may be equivalently expressed [7] as

$$
\forall(s, z) \in \mathbb{C}^{2} \text { with } \operatorname{Re} s \geq 0,|z| \leq 1, \operatorname{det}\left(s I-A-z A_{d}\right) \neq 0 .
$$

An important step has been accomplished by Chen et al. [4], who showed that interpreting the delay as a norm-bounded uncertainty is a fruitful approach to analyze delay-independent stability. This approach permits the use of techniques originated from robust stability analysis, in particular the notion of structured singular value [23]. Isolating the delay in an uncertainty block, a necessary and sufficient condition (equivalent to (10) and (11)) for delay-independent stability of system (8) is expressed as [4, Theorem 3.1]

$$
\operatorname{Re} \sigma(A)<0, \operatorname{det}\left(A+A_{d}\right) \neq 0 \text { and } \forall s \in j \mathbb{R} \backslash\{0\}, \rho\left((s I-A)^{-1} A_{d}\right)<1 .
$$

Here, $\rho$ designates the spectral radius, and is equal, in this simple case, to the structured singular value $\mu_{\Delta}\left((s I-A)^{-1} A_{d}\right)$ resulting from the coupling of the transfer $(s I-A)^{-1} A_{d}$ with an uncertainty from the set $\Delta \stackrel{\text { def }}{=}\{\delta I: \delta \in \mathbb{C},|\delta| \leq 1\}$. Analogously, (12) and (13) may be shown to be equivalent to

$$
\operatorname{Re} \sigma(A)<0 \text { and } \forall s \in j \mathbb{R}, \rho\left((s I-A)^{-1} A_{d}\right)<1 .
$$

This approach also permits to give some stability conditions for systems with uncertain matrices $A, A_{d}[4]$.

\section{Main result}

Define

$$
H(s, z) \stackrel{\text { def }}{=}\left(C+z C_{d}\right)\left(s I-A-z A_{d}\right)^{-1} B .
$$


Theorem 1. Consider the six following conditions.

Condition (A1). There exist $P, Q \in \mathbb{R}^{n \times n}$, positive definite, such that

$$
R \stackrel{\text { def }}{=}\left(\begin{array}{ccc}
A^{T} P+P A+Q+C^{T} K^{T} K C & P A_{d}+C^{T} K^{T} K C_{d} & -P B \\
A_{d}^{T} P+C_{d}^{T} K^{T} K C & -Q+C_{d}^{T} K^{T} K C_{d} & 0 \\
-B^{T} P & 0 & -I
\end{array}\right)<0 .
$$

Condition (B1). $\operatorname{Re} \sigma(A)<0$ and

$$
\min _{\substack{M \\
\text { invertible }}} \max _{s \in j \mathbb{R}}\left\|\left(\begin{array}{cc}
M & 0 \\
0 & I
\end{array}\right)\left(\begin{array}{cc}
(s I-A)^{-1} A_{d} & -(s I-A)^{-1} B \\
K\left(C(s I-A)^{-1} A_{d}+C_{d}\right) & -K C(s I-A)^{-1} B
\end{array}\right)\left(\begin{array}{cc}
M^{-1} & 0 \\
0 & I
\end{array}\right)\right\|<1 .
$$

Condition (A2). $\forall(s, z) \in \mathbb{C}^{2}$ with $\operatorname{Re} s \geq 0,|z| \leq 1, \operatorname{det}\left(s I-A-z A_{d}\right) \neq 0$ and $\|K H(s, z)\|<1$, where $H$ is given by (16).

Condition (B2). $\operatorname{Re} \sigma(A)<0$ and

$$
\max _{s \in j \mathbb{R}} \min _{\substack{M \\
\text { invertible }}}\left\|\left(\begin{array}{cc}
M & 0 \\
0 & I
\end{array}\right)\left(\begin{array}{cc}
(s I-A)^{-1} A_{d} & -(s I-A)^{-1} B \\
K\left(C(s I-A)^{-1} A_{d}+C_{d}\right) & -K C(s I-A)^{-1} B
\end{array}\right)\left(\begin{array}{cc}
M^{-1} & 0 \\
0 & I
\end{array}\right)\right\|<1 .
$$

Condition (A3). $\forall h \geq 0, \forall s \in \mathbb{C}$ with $\operatorname{Re} s \geq 0, \operatorname{det}\left(s I-A-e^{-s h} A_{d}\right) \neq 0$ and $\left\|K H\left(s, e^{-s h}\right)\right\|<1$, where $H$ is given by $(16)$.

Condition (B3). $\operatorname{Re} \sigma(A)<0$,

$$
\max _{s \in j \mathbb{R} \backslash\{0\}} \min _{\substack{M \\
\text { invertible }}}\left\|\left(\begin{array}{cc}
M & 0 \\
0 & I
\end{array}\right)\left(\begin{array}{cc}
(s I-A)^{-1} A_{d} & -(s I-A)^{-1} B \\
K\left(C(s I-A)^{-1} A_{d}+C_{d}\right) & -K C(s I-A)^{-1} B
\end{array}\right)\left(\begin{array}{cc}
M^{-1} & 0 \\
0 & I
\end{array}\right)\right\|<1,
$$

and

$$
\operatorname{det}\left(A+A_{d}\right) \neq 0 \text { and }\left\|K\left(C+C_{d}\right)\left(A+A_{d}\right)^{-1} B\right\|<1 .
$$

Then, the following implications are verified.

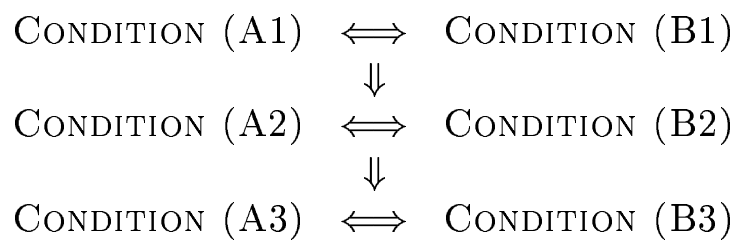

Moreover, any of the six conditions ensure global asymptotic stability of system (5), for any value $h \geq 0$ of the delay and for any nonlinearity $\psi$ fulfilling (1).

Condition (A1) must be related to (3), conditions (A2) and (A3) to (4), condition (B2) to (15) and condition (B3) to (14). Conditions (A3)/(B3) imply internal delay-independent stability property (9), and conditions (A2)/(B2) internal strong delay-independent stability property (13).

Condition (A1) is the condition for success of Lyapunov-Krasovskii method, when searching for functionals in the class (7). Condition (A3) means exactly that small gain theorem applies for any nonnegative value of the delay $h$. It is slightly weaker than condition (A2), in the same manner than delay-independent stability is slightly weaker than strong delay-independent stability.

Checking numerically conditions (B2) and (B3) requires to compute structured singular value with respect to real and complex uncertainties. Indeed,

$$
\min _{\substack{M \\
\text { invertible }}}\left\|\left(\begin{array}{cc}
M & 0 \\
0 & I
\end{array}\right)\left(\begin{array}{cc}
(s I-A)^{-1} A_{d} & -(s I-A)^{-1} B \\
K\left(C(s I-A)^{-1} A_{d}+C_{d}\right) & -K C(s I-A)^{-1} B
\end{array}\right)\left(\begin{array}{cc}
M^{-1} & 0 \\
0 & I
\end{array}\right)\right\|
$$

INRIA 
is equal [23, Theorem 11.5] to the structured singular value

$$
\mu_{\Delta}\left(\left(\begin{array}{cc}
(s I-A)^{-1} A_{d} & -(s I-A)^{-1} B \\
K\left(C(s I-A)^{-1} A_{d}+C_{d}\right) & -K C(s I-A)^{-1} B
\end{array}\right)\right),
$$

where the uncertainty set $\Delta$ is here defined as $\left\{\operatorname{diag}\{\delta I, D\}: \delta \in \mathbb{C},|\delta| \leq 1, D \in \mathbb{R}^{q \times q},\|D\| \leq 1\right\}$. This amounts to analyze the asymptotic stability of (5) replacing the delay and the nonlinearity (constrained by the growth condition) by two sources of uncertainty, see also [4, Section IV]. As quoted therein, the numerical resolution of this problem involves computing structured singular values with respect to real and complex uncertainties, and is hence in general NP-hard [3]. On the contrary, condition (A1) is a LMI and may be easily verified numerically [2], at the cost of a certain conservativity.

Remark that conditions $(\mathrm{A} 1) /(\mathrm{B} 1)$ and $(\mathrm{A} 2) /(\mathrm{B} 2)$ are robust with respect to parametric perturbations of the matrices $A, A_{d}, B, C, C_{d}$, contrary to conditions (A3)/(B3). Indeed, in this parameters space endowed with the product topology induced by the spectral norm, the sets of those systems fulfilling (A1)/(B1) or (A2)/(B2) are open. One shows easily that the set of the systems fulfilling (A2)/(B2) is precisely the interior of the set of the systems fulfilling (A3)/(B3).

Two particular cases are worth noting. First, in the delay-free case $\left(A_{d}=0, C_{d}=0\right)$, Theorem 1 simply expresses that asymptotic stability of system (2) under assumption (1) is a consequence of small gain theorem, expressed equivalently by (3) or (4). The equivalence of these two forms is a by-product of Kalman-Yakubovich-Popov lemma, which is a key ingredient of the proof of Theorem 1.

In the linear, or uncertainty-free, case $(K=0)$, the equivalence of conditions (A3) and (B3) is exactly the characterization of delay-independent stability given by Chen et al. [4, Theorem 3.1], and conditions (A2), (B2) characterize strong delay-independent stability. Condition (A1), that is feasability of the LMI

$$
\exists P=P^{T}>0, Q=Q^{T}>0,\left(\begin{array}{cc}
A^{T} P+P A+Q & P A_{d} \\
A_{d}^{T} P & -Q
\end{array}\right)<0,
$$

is hence proved to be sufficient for strong delay-independent stability of the linear system (8). To our knowledge, this is the first comparison result linking precisely Lyapunov-Krasovskii approach and delay-independent stability.

\section{Applications}

\subsection{Examples of nonconservativity of Lyapunov-Krasovskii method}

When the min and the max may be interverted in conditions (B1) and (B2), then checking condition (A1) gives a complete answer on the effectiveness of (A2), a condition very close from (A3), the optimal one (which however is not robust wrt parametric uncertainties, as indicated above after Theorem 1). This principle permits to exhibit some classes of examples where (A1) and (A2) are equivalent.

The first example concerns the linear system (8).

Theorem 2. Suppose $A A_{d}=A_{d} A$. System (8) is strongly delay-independently stable [15] if and only if the following LMI is feasible

$$
\exists P=P^{T}>0, Q=Q^{T}>0,\left(\begin{array}{cc}
A^{T} P+P A+Q & P A_{d} \\
A_{d}^{T} P & -Q
\end{array}\right)<0 .
$$

Proof. When $A$ and $A_{d}$ commute and have simple eigenvalues, they are diagonalizable in a common basis [5]. Taking for $M$ the corresponding similarity transformation, one sees that both expressions in conditions (B1), (B2) are equal to $\max \left\{\rho\left((s I-A)^{-1} A_{d}\right): s \in j \mathbb{R}\right\}$, whence the equivalence between 
conditions (A1) and (A2). When either $A$ or $A_{d}$ has eigenvalues with multiplicity more than one, one may find arbitrarily small matrices $\tilde{A}$ such that $A+\tilde{A}$ and $A_{d}+\tilde{A}$ commute (i.e. $\tilde{A}$ and $A-A_{d}$ commute) and have simple eigenvalues. It then suffices to apply the previous argument, and to make $\tilde{A} \rightarrow 0$.

The second example concerns the following class of systems with undelayed output. Let $A=a I, a \in$ $\mathbb{R}$, and consider

$$
\dot{x}=a x+A_{d} x(t-h)-B \psi(t, y), y=C x,
$$

where $\psi$ fulfills (1).

Theorem 3. The two following conditions are equivalent and ensure asymptotic stability of system (19), for any $h \geq 0$ and any nonlinearity $\psi$ fulfilling (1).

- There exist $P=P^{T}>0, Q=Q^{T}>0$ such that

$$
\left(\begin{array}{ccc}
2 a P+Q+C^{T} K^{T} K C & P A_{d} & -P B \\
A_{d}^{T} P & -Q & 0 \\
-B^{T} P & 0 & -I
\end{array}\right)<0 .
$$

- For all $(s, z) \in \mathbb{C}^{2}$ with $\operatorname{Re} s \geq 0,|z| \leq 1$, $\operatorname{det}\left((s-a) I-z A_{d}\right) \neq 0$ and $\left\|K C\left((s-a) I-z A_{d}\right)^{-1} B\right\|<$ 1.

Proof. As

$$
\begin{gathered}
\left(\begin{array}{cc}
M & 0 \\
0 & I
\end{array}\right)\left(\begin{array}{cc}
(s I-A)^{-1} A_{d} & -(s I-A)^{-1} B \\
K C(s I-A)^{-1} A_{d} & -K C(s I-A)^{-1} B
\end{array}\right)\left(\begin{array}{cc}
M^{-1} & 0 \\
0 & I
\end{array}\right) \\
=(s-a)^{-1}\left(\begin{array}{cc}
M & 0 \\
0 & I
\end{array}\right)\left(\begin{array}{cc}
A_{d} & -B \\
K C A_{d} & -K C B
\end{array}\right)\left(\begin{array}{cc}
M^{-1} & 0 \\
0 & I
\end{array}\right)
\end{gathered}
$$

the complex matrix which realizes the minimum of

$$
M \mapsto\left\|\left(\begin{array}{cc}
M & 0 \\
0 & I
\end{array}\right)\left(\begin{array}{cc}
(s I-A)^{-1} A_{d} & -(s I-A)^{-1} B \\
K C(s I-A)^{-1} A_{d} & -K C(s I-A)^{-1} B
\end{array}\right)\left(\begin{array}{cc}
M^{-1} & 0 \\
0 & I
\end{array}\right)\right\|
$$

is independent of $s$. This permits to reverse the order of max and min in condition (B2), which is then shown to be equivalent to (B1). Conditions (A1) and (A2) are thus equivalent.

\subsection{Systems with commensurate delays}

We establish here a generalization of Theorem 1 to the following class of systems:

$$
\dot{x}=\sum_{l=0}^{\bar{l}} A_{l} x(t-l h)-B \psi(t, y), y=\sum_{l=0}^{\bar{l}} C_{l} x(t-l h)
$$

where $\bar{l} \in \mathbb{N}$ and $A_{l} \in \mathbb{R}^{n \times n}, C_{l} \in \mathbb{R}^{p \times n}$ for $l=\overline{0, \bar{l}}, B \in \mathbb{R}^{n \times q}$. The idea to handle system (20), is to transform it into a new, augmented, system having a unique delay, in order to be able to apply the previous ideas of quadratic Lyapunov-Krasovskii functionals. More precisely, defining

$$
\mathcal{X}(t) \stackrel{\text { def }}{=}\left(\begin{array}{c}
x(t) \\
x(t-h) \\
\vdots \\
x(t-(\bar{l}-1) h)
\end{array}\right), \Psi(t, \mathcal{Y}) \stackrel{\text { def }}{=}\left(\begin{array}{c}
\psi\left(t, \mathcal{Y}_{1}\right) \\
\psi\left(t-h, \mathcal{Y}_{2}\right) \\
\vdots \\
\psi\left(t-(\bar{l}-1) h, \mathcal{Y}_{\bar{l}}\right)
\end{array}\right),
$$


one has, when $x$ is solution of system (20):

$$
\dot{\mathcal{X}}=\mathcal{A} \mathcal{X}+\mathcal{A}_{d} \mathcal{X}(t-\bar{l} h)-\mathcal{B} \Psi(t, \mathcal{Y}), \mathcal{Y}=\mathcal{C X}+\mathcal{C}_{d} \mathcal{X}(t-\bar{l} h)
$$

where $(\otimes$ denotes Kronecker product)

$$
\begin{gathered}
\mathcal{A} \stackrel{\text { def }}{=} \sum_{l=0}^{\bar{l}} J^{l} \otimes A_{l}, \mathcal{A}_{d} \stackrel{\text { def }}{=} \sum_{l=0}^{\bar{l}} J^{(\bar{l}-l) T} \otimes A_{l}, \mathcal{B} \stackrel{\text { def }}{=} I_{l} \otimes B, \\
\mathcal{C} \stackrel{\text { def }}{=} \sum_{l=0}^{\bar{l}} J^{l} \otimes C_{l}, \mathcal{C}_{d} \stackrel{\text { def }}{=} \sum_{l=0}^{\bar{l}} J^{(\bar{l}-l) T} \otimes C_{l}, \\
J \in \mathbb{R}^{\bar{l} \times \bar{l}}, J_{i j} \stackrel{\text { def }}{=} 1 \text { if } i+1=j, 0 \text { otherwise }, i, j=\overline{1, \bar{l}}
\end{gathered}
$$

and

$$
\forall t \geq 0, \forall \mathcal{Y} \in \mathbb{R}^{p \bar{l}},\|\Psi(t, \mathcal{Y})\| \leq\|\mathcal{K} \mathcal{Y}\|, \text { where } \mathcal{K} \stackrel{\text { def }}{=} I_{\bar{l}} \otimes K
$$

The previous transformation offers the possibility to use the approach adopted in Section 2, searching for quadratic Lyapunov-Krasovskii functional for system (21) in the class

$$
V(\phi) \stackrel{\text { def }}{=} \phi^{T}(0) \mathcal{P} \phi(0)+\int_{-\bar{l} h}^{0} \phi^{T}(\tau) \mathcal{Q} \phi(\tau) d \tau
$$

defined for any $\phi \in \mathcal{C}\left([-\bar{l} h, 0] ; \mathbb{R}^{n \bar{l}}\right)$.

The trajectories of system (20) may be obtained as projections of trajectories of system (21): asymptotic stability of (21) hence implies asymptotic stability of (20). An interesting feature is that the stability property defined by condition (A2) is indeed equivalent for systems (20) and (21). This gives rise to the following result (in which the analogs of conditions (A3), (B3) are omitted for sake of space). Define

$$
H(s, z) \stackrel{\text { def }}{=}\left(\sum_{l=0}^{\bar{l}} z^{l} C_{l}\right)\left(s I-\sum_{l=0}^{\bar{l}} z^{l} A_{l}\right)^{-1} B
$$

Theorem 4. Consider the four following conditions.

Condition $(\mathcal{A} 1)$. There exist $\mathcal{P}, \mathcal{Q} \in \mathbb{R}^{n \bar{l} \times n \bar{l}}$, positive definite, such that

$$
\mathcal{R} \stackrel{\operatorname{def}}{=}\left(\begin{array}{ccc}
\mathcal{A}^{T} \mathcal{P}+\mathcal{P} \mathcal{A}+\mathcal{Q}+\mathcal{C}^{T} \mathcal{K}^{T} \mathcal{K C} & \mathcal{P} \mathcal{A}_{d}+\mathcal{C}^{T} \mathcal{K}^{T} \mathcal{K} \mathcal{C}_{d} & -\mathcal{P} \mathcal{B} \\
\mathcal{A}_{d}^{T} \mathcal{P}+\mathcal{C}_{d}^{T} \mathcal{K}^{T} \mathcal{K C} & -\mathcal{Q}+\mathcal{C}_{d}^{T} \mathcal{K}^{T} \mathcal{K} \mathcal{C}_{d} & 0 \\
-\mathcal{B}^{T} \mathcal{P} & 0 & -I
\end{array}\right)<0
$$

Condition $(\mathcal{B} 1) . \operatorname{Re} \sigma\left(A_{0}\right)<0$ and

$$
\min _{\substack{M \\
\text { invertible }}} \max _{s \in j \mathbb{R}}\left\|\left(\begin{array}{cc}
M & 0 \\
0 & I
\end{array}\right)\left(\begin{array}{cc}
(s I-\mathcal{A})^{-1} \mathcal{A}_{d} & -(s I-\mathcal{A})^{-1} \mathcal{B} \\
\mathcal{K}\left(\mathcal{C}(s I-\mathcal{A})^{-1} \mathcal{A}_{d}+\mathcal{C}_{d}\right) & -\mathcal{K} \mathcal{C}(s I-\mathcal{A})^{-1} \mathcal{B}
\end{array}\right)\left(\begin{array}{cc}
M^{-1} & 0 \\
0 & I
\end{array}\right)\right\|<1 .
$$

Condition $(\mathcal{A} 2) . \forall(s, z) \in \mathbb{C}^{2}$ with $\operatorname{Re} s \geq 0,|z| \leq 1, \operatorname{det}\left(s I-\sum_{l=0}^{\bar{l}} z^{l} A_{l}\right) \neq 0$ and $\|K H(s, z)\|<1$, where $H$ is given by (24).

Condition $(\mathcal{B} 2) . \operatorname{Re} \sigma\left(A_{0}\right)<0$ and

$$
\max _{s \in j \mathbb{R}} \min _{\substack{M \\
\text { invertible }}}\left\|\left(\begin{array}{cc}
M & 0 \\
0 & I
\end{array}\right)\left(\begin{array}{cc}
(s I-\mathcal{A})^{-1} \mathcal{A}_{d} & -(s I-\mathcal{A})^{-1} \mathcal{B} \\
\mathcal{K}\left(\mathcal{C}(s I-\mathcal{A})^{-1} \mathcal{A}_{d}+\mathcal{C}_{d}\right) & -\mathcal{K} \mathcal{C}(s I-\mathcal{A})^{-1} \mathcal{B}
\end{array}\right)\left(\begin{array}{cc}
M^{-1} & 0 \\
0 & I
\end{array}\right)\right\|<1 .
$$

$\mathrm{RR} \mathrm{n}^{\circ} 3969$ 
Then, the following implications are verified.

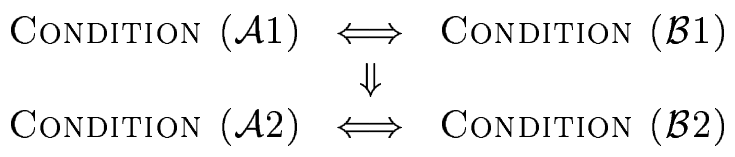

Moreover, any of the four conditions ensure global asymptotic stability of system (20), for any value $h \geq 0$ of the delay and for any nonlinearity $\psi$ fulfilling (1).

Proof. In view of Theorem 1, it only remains to prove that

$$
\forall(s, z) \in \mathbb{C}^{2} \text { with } \operatorname{Re} s \geq 0,|z| \leq 1, \operatorname{det}\left(s I-\sum_{l=0}^{\bar{l}} z^{l} A_{l}\right) \neq 0 \text { and }\|K H(s, z)\|<1 \Leftrightarrow
$$

$\forall(s, z) \in \mathbb{C}^{2}$ with $\operatorname{Re} s \geq 0,|z| \leq 1, \operatorname{det}\left(s I-\mathcal{A}-z \mathcal{A}_{d}\right) \neq 0$ and $\left\|\mathcal{K}\left(\mathcal{C}+z \mathcal{C}_{d}\right)\left(s I-\mathcal{A}-z \mathcal{A}_{d}\right)^{-1} \mathcal{B}\right\|<1$

Define, for any $z \in \mathbb{C}$,

$$
r=r(\bar{l}) \stackrel{\text { def }}{=} e^{-\frac{2 i \pi}{\bar{l}}}, N=N(z, \bar{l}) \stackrel{\text { def }}{=}\left(N_{i j}\right), \text { where } N_{i j} \stackrel{\text { def }}{=}\left(r^{j-1} z\right)^{i-1}, i, j=\overline{1, \bar{l}} .
$$

The properties gathered in the following auxiliary lemma, whose proof is left to the reader, will be used.

Lemma 5. The following formulae are true.

$$
\begin{gathered}
r^{\bar{l}}=1, \\
|z|=1 \Rightarrow N^{*} N=I, \\
\forall l=\overline{0, \bar{l}}, \quad J^{l}+z^{\bar{l}} J^{(\bar{l}-l) T}=N \operatorname{diag}\left\{z^{l},(r z)^{l}, \ldots,\left(r^{\bar{l}-1} z\right)^{l}\right\} N^{-1} .
\end{gathered}
$$

The key point in the last formula is that $r$ and $N$ do not depend upon $l$. Using Lemma 5 , one shows that

$$
\begin{aligned}
\operatorname{det}\left(s I-\mathcal{A}-z^{\bar{l}} \mathcal{A}_{d}\right) & =\operatorname{det}\left(s I-\sum_{l=0}^{\bar{l}}\left(J^{l}+z^{\bar{l}} J^{(\bar{l}-l) T}\right) \otimes A_{l}\right) \\
& =\operatorname{det}\left(s I-\sum_{l=0}^{\bar{l}} \operatorname{diag}\left\{z^{l} A_{l},(r z)^{l} A_{l}, \ldots,\left(r^{\bar{l}-1} z\right)^{l} A_{l}\right\}\right) \\
& =\prod_{l^{\prime}=0}^{\bar{l}-1} \operatorname{det}\left(s I-\sum_{l=0}^{\bar{l}}\left(r^{l^{\prime}} z\right)^{l} A_{l}\right)
\end{aligned}
$$

As $|r|=1$ and $z \mapsto z^{\bar{l}}$ maps the unit ball onto itself, it is thus proved that

$$
\begin{aligned}
& \forall(s, z) \in \mathbb{C}^{2} \text { with } \operatorname{Re} s \geq 0,|z| \leq 1, \operatorname{det}\left(s I-\sum_{l=0}^{\bar{l}} z^{l} A_{l}\right) \neq 0 \Leftrightarrow \\
& \forall(s, z) \in \mathbb{C}^{2} \text { with } \operatorname{Re} s \geq 0,|z| \leq 1, \operatorname{det}\left(s I-\mathcal{A}-z \mathcal{A}_{d}\right) \neq 0 .
\end{aligned}
$$


Also,

$$
\begin{aligned}
\mathcal{K}\left(\mathcal{C}+z^{\bar{l}} \mathcal{C}_{d}\right)\left(s I-\mathcal{A}-z^{\bar{l}} \mathcal{A}_{d}\right)^{-1} \mathcal{B} \\
=\left(I_{\bar{l}} \otimes K\right)\left(N \otimes I_{p}\right)\left[\sum_{l=0}^{\bar{l}} \operatorname{diag}\left\{z^{l} C_{l},(r z)^{l} C_{l}, \ldots,\left(r^{\bar{l}-1} z\right)^{l} C_{l}\right\}\right] \\
\\
\quad\left[s I-\sum_{l=0}^{\bar{l}} \operatorname{diag}\left\{z^{l} A_{l},(r z)^{l} A_{l}, \ldots,\left(r^{\bar{l}-1} z\right)^{l} A_{l}\right\}\right]^{-1}\left(N^{-1} \otimes I_{q}\right)\left(I_{\bar{l}} \otimes B\right) \\
=\left(N \otimes I_{q}\right) \operatorname{diag}\left\{K H(s, z), K H(s, r z), \ldots, K H\left(s, r^{\bar{l}} z\right)\right\}\left(N^{-1} \otimes I_{q}\right) .
\end{aligned}
$$

Thus, using the second property in Lemma 5 and the fact that $|r|=1$, one gets that

$$
\begin{aligned}
\forall(s, z) \in \mathbb{C}^{2} \text { with } \operatorname{Re} s & \geq 0,|z|=1,\|K H(s, z)\|<1 \Leftrightarrow \\
\forall(s, z) & \in \mathbb{C}^{2} \text { with } \operatorname{Re} s \geq 0,|z|=1,\left\|\mathcal{K}\left(\mathcal{C}+z \mathcal{C}_{d}\right)\left(s I-\mathcal{A}-z \mathcal{A}_{d}\right)^{-1} \mathcal{B}\right\|<1 .
\end{aligned}
$$

The same property is extended to $|z| \leq 1$ using maximum modulus principle and the analyticity of the maps $z \mapsto\left(s I-\mathcal{A}-z \mathcal{A}_{d}\right)^{-1}, z \mapsto\left(s I-\sum_{l=0}^{\bar{l}} z^{l} A_{l}\right)^{-1}$ in the unit ball, for any $s \in \mathbb{C}$ such that $\operatorname{Re} s \geq 0$. This concludes the proof of formula (26) and of Theorem 4 .

\section{Proof of Theorem 1}

Both continuous-time and discrete-time versions of Kalman-Yakubovich-Popov lemma will be used ${ }^{1}$, under the following form [17]. Denote $\overline{\mathbb{R}} \stackrel{\text { def }}{=} \mathbb{R} \cup\{\infty\}$. Let $A \in \mathbb{R}^{n \times n}, B \in \mathbb{R}^{n \times p}, M=M^{T} \in$ $\mathbb{R}^{(n+p) \times(n+p)}$ with

$$
\left(\begin{array}{ll}
I_{n} & 0_{n \times p}
\end{array}\right) M\left(\begin{array}{c}
I_{n} \\
0_{p \times n}
\end{array}\right) \geq 0 .
$$

Lemma 6 (Continuous-time positive real lemma). For any matrix $M$ fulfilling (27), the following two statements are equivalent:

(i) There exists $P=P^{T} \in \mathbb{R}^{n \times n}$, positive definite, such that

$$
M+\left(\begin{array}{cc}
A^{T} P+P A & P B \\
B^{T} P & 0_{p}
\end{array}\right)<0 .
$$

(ii) $\operatorname{Re} \sigma(A)<0$ and, for any $s \in j \overline{\mathbb{R}}$,

$$
\left(\begin{array}{c}
\left(s I_{n}-A\right)^{-1} B \\
I_{p}
\end{array}\right)^{*} M\left(\begin{array}{c}
\left(s I_{n}-A\right)^{-1} B \\
I_{p}
\end{array}\right)<0 .
$$

Lemma 7 (Discrete-time positive real lemma). For any matrix $M$ fulfilling (27), the following two statements are equivalent:

\footnotetext{
${ }^{1}$ The continuous-time version is due to Kalman [9] and Yakubovich [21], the discrete-time to Szegö and Kalman [19].
} 
(i) There exists $Q=Q^{T} \in \mathbb{R}^{n \times n}$, positive definite, such that

$$
M+\left(\begin{array}{cc}
A^{T} Q A-Q & A^{T} Q B \\
B^{T} Q A & B^{T} Q B
\end{array}\right)<0 .
$$

(ii) $|\sigma(A)|<1$ and, for any $z \in \mathbb{C}$ with $|z|=1$,

$$
\left(\begin{array}{c}
\left(z I_{n}-A\right)^{-1} B \\
I_{p}
\end{array}\right)^{*} M\left(\begin{array}{c}
\left(z I_{n}-A\right)^{-1} B \\
I_{p}
\end{array}\right)<0
$$

It is straightforward, comparing conditions (B1) and (B2), and conditions (A2) and (A3), to demonstrate the vertical implications in (18). We now show the three horizontal equivalences.

- Suppose condition (A1) holds, that is: there exist $P, Q \in \mathbb{R}^{n \times n}$ positive definite such that

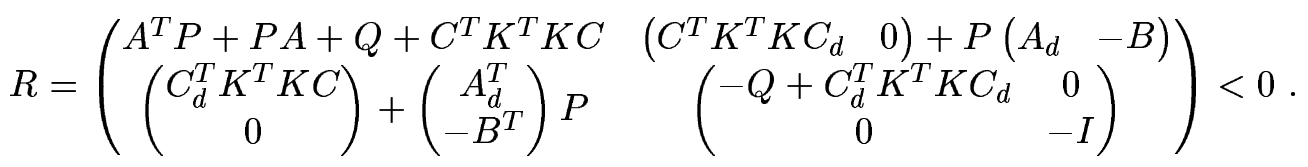

Using Lemma 6 , the latter is equivalent to: $\operatorname{Re} \sigma(A)<0$ and

$$
\begin{aligned}
\exists Q=Q^{T}>0, \forall s & \in j \mathbb{R},\left(\begin{array}{cc}
-Q+C_{d}^{T} K^{T} K C_{d} & 0 \\
0 & -I
\end{array}\right)+\left(\begin{array}{c}
C_{d}^{T} K^{T} K C \\
0
\end{array}\right) S\left(\begin{array}{c}
A_{d}^{T} \\
-B^{T}
\end{array}\right)^{T} \\
& +\left(\begin{array}{c}
A_{d}^{T} \\
-B^{T}
\end{array}\right) S^{*}\left(\begin{array}{c}
C_{d}^{T} K^{T} K C \\
0
\end{array}\right)^{T}+\left(\begin{array}{c}
A_{d}^{T} \\
-B^{T}
\end{array}\right) S^{*}\left(Q+C^{T} K^{T} K C\right) S\left(\begin{array}{c}
A_{d}^{T} \\
-B^{T}
\end{array}\right)^{T}<0
\end{aligned}
$$

where the irrelevant infinite values of $s$ have been removed and, for sake of simplicity, one has written $S=S(s) \stackrel{\text { def }}{=}(s I-A)^{-1}$. Simple computation shows that the previous inequality writes:

$$
\left(\begin{array}{cc}
S A_{d} & -S B \\
K\left(C S A_{d}+C_{d}\right) & -K C S B
\end{array}\right)^{*}\left(\begin{array}{cc}
Q & 0 \\
0 & I
\end{array}\right)\left(\begin{array}{cc}
S A_{d} & -S B \\
K\left(C S A_{d}+C_{d}\right) & -K C S B
\end{array}\right)<\left(\begin{array}{cc}
Q & 0 \\
0 & I
\end{array}\right) .
$$

The fact that the sets $\left\{Q: Q=Q^{T}>0\right\}$ and $\left\{M^{*} M: M\right.$ invertible $\}$ are identical yields the equivalence between conditions (A1) and (B1).

- Let us now show the equivalence between conditions (A2) and (B2). In both cases $A$ is Hurwitz, so the map

$$
s \mapsto\left(\begin{array}{cc}
(s I-A)^{-1} A_{d} & -(s I-A)^{-1} B \\
K\left(C(s I-A)^{-1} A_{d}+C_{d}\right) & -K C(s I-A)^{-1} B
\end{array}\right)
$$

is analytic in $\{s \in \mathbb{C}: \operatorname{Re} s \geq 0\}$, and the maximum on this set of the map

$$
s \mapsto \min _{\substack{M \\
\text { invertible }}}\left\|\left(\begin{array}{cc}
M & 0 \\
0 & I
\end{array}\right)\left(\begin{array}{cc}
(s I-A)^{-1} A_{d} & -(s I-A)^{-1} B \\
K\left(C(s I-A)^{-1} A_{d}+C_{d}\right) & -K C(s I-A)^{-1} B
\end{array}\right)\left(\begin{array}{cc}
M^{-1} & 0 \\
0 & I
\end{array}\right)\right\|
$$

is hence achieved on the imaginary axis. Thus, condition (B2) is equivalent to:

$\operatorname{Re} \sigma(A)<0$ and $\forall s \in \mathbb{C}$ with $\operatorname{Re} s \geq 0, \exists M$ invertible,

$$
\left(\begin{array}{cc}
S A_{d} & -S B \\
K\left(C S A_{d}+C_{d}\right) & -K C S B
\end{array}\right)^{*}\left(\begin{array}{cc}
M^{*} M & 0 \\
0 & I
\end{array}\right)\left(\begin{array}{cc}
S A_{d} & -S B \\
K\left(C S A_{d}+C_{d}\right) & -K C S B
\end{array}\right)<\left(\begin{array}{cc}
M^{*} M & 0 \\
0 & I
\end{array}\right)
$$


that is, replacing $M^{*} M$ by $Q=Q^{T}>0$ and developing:

$\operatorname{Re} \sigma(A)<0$ and $\forall s \in \mathbb{C}$ with $\operatorname{Re} s \geq 0, \exists Q=Q^{T}>0,\left(\begin{array}{cc}A_{d}^{T} S^{*} Q S A_{d}-Q & -A_{d}^{T} S^{*} Q S B \\ -B^{T} S^{*} Q S A_{d} & B^{T} S^{*} Q S B\end{array}\right)$

$$
+\left(\begin{array}{cc}
\left(A_{d}^{T} S^{*} C^{T}+C_{d}^{T}\right) K^{T} K\left(C S A_{d}+C_{d}\right) & -\left(A_{d}^{T} S^{*} C^{T}+C_{d}^{T}\right) K^{T} K C S B \\
-B^{T} S^{*} C^{T} K^{T} K\left(C S A_{d}+C_{d}\right) & B^{T} S^{*} C^{T} K^{T} K C S B-I
\end{array}\right)<0 .
$$

Applying now Lemma 7, one first deduces that: $\forall(s, z) \in \mathbb{C}^{2}$ with $\operatorname{Re} s \geq 0,|z| \geq 1$,

$$
0 \neq \operatorname{det}\left(z I-S A_{d}\right)=\operatorname{det}\left(z I-(s I-A)^{-1} A_{d}\right)=\frac{1}{z^{n} \operatorname{det}(s I-A)} \operatorname{det}\left(s I-A-z^{-1} A_{d}\right) .
$$

Changing $z$ into $z^{-1}$ leads to:

$$
\forall(s, z) \in \mathbb{C}^{2} \text { with } \operatorname{Re} s \geq 0,|z| \leq 1, \operatorname{det}\left(s I-A-z A_{d}\right) \neq 0 .
$$

On the other hand, application of Lemma 7 also yields: $\forall(s, z) \in \mathbb{C}^{2}$ with $\operatorname{Re} s \geq 0,|z|=1$,

$$
\begin{aligned}
I> & B^{T} S^{*}\left(z^{*} I-A_{d}^{T} S^{*}\right)^{-1}\left(A_{d}^{T} S^{*} C^{T}+C_{d}^{T}\right) K^{T} K\left(C S A_{d}+C_{d}\right)\left(z I-S A_{d}\right)^{-1} S B \\
& +B^{T} S^{*} C^{T} K^{T} K\left(C S A_{d}+C_{d}\right)\left(z I-S A_{d}\right)^{-1} S B \\
& +B^{T} S^{*}\left(z^{*} I-A_{d}^{T} S^{*}\right)^{-1}\left(A_{d}^{T} S^{*} C^{T}+C_{d}^{T}\right) K^{T} K C S B+B^{T} S^{*} C^{T} K^{T} K C S B,
\end{aligned}
$$

that is

$$
\begin{aligned}
I & >B^{T} S^{*}\left[\left(z^{*} I-A_{d}^{T} S^{*}\right)^{-1}\left(A_{d}^{T} S^{*} C^{T}+C_{d}^{T}\right)+C^{T}\right] K^{T} K\left[C+\left(C S A_{d}+C_{d}\right)\left(z I-S A_{d}\right)^{-1}\right] S B \\
& =B^{T} S^{*}\left(z^{*} I-A_{d}^{T} S^{*}\right)^{-1}\left(z^{*} C^{T}+C_{d}^{T}\right) K^{T} K\left(z C+C_{d}\right)\left(z I-S A_{d}\right)^{-1} S B .
\end{aligned}
$$

Now, $\left(z I-S A_{d}\right)^{-1} S=\left(z S\left(S^{-1}-z^{-1} A_{d}\right)\right)^{-1} S=z^{-1}\left(s I-A-z^{-1} A_{d}\right)^{-1}$, so one deduces, changing $z$ into $z^{-1}$, that:

$$
\forall(s, z) \in \mathbb{C}^{2} \text { with } \operatorname{Re} s \geq 0,|z|=1, H(s, z)^{*} K^{T} K H(s, z)<I .
$$

Using maximum modulus principle, this gives (A2). Thus, (B2) implies (A2). The converse property is obtained using the converse of Lemma 7.

- Last, one proves the equivalence between conditions (A3) and (B3). The hypothesis $\operatorname{Re} \sigma(A)<0$ is indeed contained in both assertions, see [10,6]. Using maximum modulus principle, discrete-version of Kalman-Yakubovich-Popov lemma, and again maximum modulus principle, as was done for the equivalence between (A2) and (B2), one shows that, if $\operatorname{Re} \sigma(A)<0$, then the assertion

$$
\max _{s \in j \mathbb{R} \backslash\{0\}} \min _{\substack{M \\
\text { invertible }}}\left\|\left(\begin{array}{cc}
M & 0 \\
0 & I
\end{array}\right)\left(\begin{array}{cc}
(s I-A)^{-1} A_{d} & -(s I-A)^{-1} B \\
K\left(C(s I-A)^{-1} A_{d}+C_{d}\right) & -K C(s I-A)^{-1} B
\end{array}\right)\left(\begin{array}{cc}
M^{-1} & 0 \\
0 & I
\end{array}\right)\right\|<1
$$

is equivalent to

$$
\forall(s, z) \in j \mathbb{R} \times \mathbb{C} \text { with } s \neq 0,|z|=1, \operatorname{det}\left(s I-A-z A_{d}\right) \neq 0 \text { and }\|K H(s, z)\|<1,
$$

which is itself equivalent to:

$$
\forall h \geq 0, \forall s \in j \mathbb{R} \backslash\{0\}, \operatorname{det}\left(s I-A-e^{-s h} A_{d}\right) \neq 0 \text { and }\left\|K H\left(s, e^{-s h}\right)\right\|<1 .
$$

The previous assertion may be extended immediatly to any $s \in \mathbb{C} \backslash\{0\}$ with $\operatorname{Re} s \geq 0$ by maximum modulus principle. It remains to examine the case $s=0$, which is straightforward. This concludes the proof of Theorem 1. 
Remark 8. It is also possible to deduce directly condition (A2) from condition (A1). One may apply discrete-time version of Kalman-Yakubovich-Popov lemma (Lemma 7) to (28), leading directly to condition (A1). A benefit of this method is to enlighten the fact that matrix P (resp. Q) in condition (A1) is naturally linked with the continuous-time (resp. discrete-time) frequency domain variable s (resp. z) in condition (A2).

Alternatively, one may remark that, for any $(s, z) \in \mathbb{C}^{2}$ :

$$
\begin{gathered}
\left(\begin{array}{c}
-\left(s I-A-z A_{d}\right)^{-1} B \\
-z\left(s I-A-z A_{d}\right)^{-1} B \\
I
\end{array}\right){ }^{*}\left(\begin{array}{ccc}
C^{T} K^{T} K C & C^{T} K^{T} K C_{d} & 0 \\
C_{d}^{T} K^{T} K C & C_{d}^{T} K^{T} K C_{d} & 0 \\
0 & 0 & -I
\end{array}\right)\left(\begin{array}{c}
-\left(s I-A-z A_{d}\right)^{-1} B \\
-z\left(s I-A-z A_{d}\right)^{-1} B \\
I
\end{array}\right) \\
\left.=(K H(s, z))^{*}\right)(K H(s, z))-I \\
\left(\begin{array}{c}
-\left(s I-A-z A_{d}\right)^{-1} B \\
-z\left(s I-A-z A_{d}\right)^{-1} B \\
I
\end{array}\right)\left(\begin{array}{ccc}
A^{T} P+P A+Q & P A_{d} & -P B \\
A_{d}^{T} P & -Q & 0 \\
-B^{T} P & 0 & 0
\end{array}\right)\left(\begin{array}{c}
-\left(s I-A-z A_{d}\right)^{-1} B \\
-z\left(s I-A-z A_{d}\right)^{-1} B \\
I
\end{array}\right) \\
=B^{T}\left(s^{*} I-A^{T}-z^{*} A_{d}^{T}\right)^{-1}\left(\left(1-z^{*} z\right) Q+\left(s+s^{*}\right) P\right)\left(s I-A-z A_{d}\right)^{-1} B
\end{gathered}
$$

from which the implication $(\mathrm{A} 1) \Rightarrow(\mathrm{A} 2)$ is deduced immediatly. This latter proof is reminiscent of techniques used to prove the "easy" sense of Kalman-Yakubovich-Popov lemma ((i) $\Rightarrow$ (ii) in Lemmas 6 and 7). The equivalence between conditions (A1) and (A2) would lead to a "two frequency-domain variables" positive real lemma, a result which is in general false, due to the difference between conditions (B1) and (B2).

Also, left- and right-multiplicating $R$ as given in condition (A1) by $\left(\begin{array}{ccc}I & z^{*} I & 0 \\ 0 & 0 & I\end{array}\right)$ and its conjugate gives:

$$
\forall z \in \mathbb{C},|z| \leq 1,\left(\begin{array}{cc}
\left(A^{T}+z^{*} A_{d}^{T}\right) P+P\left(A+z A_{d}\right)+\left(C^{T}+z^{*} C_{d}^{T}\right) K^{T} K\left(C+z C_{d}\right) & -P B \\
-B^{T} P & -I
\end{array}\right)<0,
$$

to be compared with (3).

\section{References}

[1] P.-A. Bliman, Lyapunov-Krasovskii functionals and frequency domain: delay-independent absolute stability criteria for delay systems, submitted (2000)

[2] S. Boyd, L. El Ghaoui, E. Feron, V. Balakrishnan, Linear matrix inequalities in system and control theory, SIAM Studies in Applied Mathematics vol. 15 (1994)

[3] R.P. Braatz, P.M. Young, J.C. Doyle, M. Morari, Computational complexity of $\mu$ calculation, IEEE Trans. Automat. Contr. 39 no 5 (1994) 1000-1002

[4] J. Chen, H.A. Latchman, Frequency sweeping tests for stability independent of delay, IEEE Trans. Automat. Control 40 no 9 (1995) 1640-1645

[5] F.R. Gantmakher, Theory of matrices, Chelsea Publishing co (1959)

[6] J.K. Hale, E.F. Infante, F.S.P. Tsen, Stability in linear delay equations, J. Math. Anal. Appl. 115 (1985) 533-555

[7] D. Hertz, E.I. Jury, E. Zeheb, Stability independent and dependent of delay for delay differential systems, J. Franklin Institute 318 no 3 (1984) 143-150

INRIA 
[8] Y.-P. Huang, K. Zhou, Robust control of uncertain time delay systems, Proc. of 38th Conf. on Decision and Control, Phoenix, Arizona (1999) 1130-1135

[9] R.E. Kalman, Lyapunov functions for the problem of Lur'e in automatic control, Proc. Nat. Acad. Sci. 49 (1963) 201-205

[10] E.W. Kamen, On the relationship between zero criteria for two-variable polynomials and asymptotic stability of delay differential equations, IEEE Trans. Automat. Control 25 no 5 (1980) 983984

[11] E.W. Kamen, Linear systems with commensurate time delays: stability and stabilization independent of delay. IEEE Trans. Automat. Control 27 no 2 (1982) 367-375

[12] E.W. Kamen, Correction to "Linear systems with commensurate time delays: stability and stabilization independent of delay", IEEE Trans. Automat. Control 28 no 2 (1983) 248-249

[13] V.L. Kharitonov, Robust stability analysis of time delay systems: a survey, Proc. IFAC Syst. Struct. Contr. (1998)

[14] N.N. Krasovskii, Stability of motion, Stanford University Press (1963)

[15] S.-I. Niculescu, J.-M. Dion, L. Dugard, H. Li, Asymptotic stability sets for linear systems with commensurable delays: a matrix pencil approach, IEEE/IMACS CESA'96, Lille, France (1996)

[16] S.-I. Niculescu, E.I. Verriest, L. Dugard, J.-M. Dion, Stability and robust stability of time-delay systems: a guided tour, Stability and control of time-delay systems, Lecture Notes in Control and Inform. Sci. 228, Springer, London (1998) 1-71

[17] A. Rantzer, On the Kalman-Yakubovich-Popov lemma, Syst. Contr. Lett. 28 no 1 (1996) 7-10

[18] I.W. Sandberg, A frequency domain condition for stability of feedback systems containing a single time-varying nonlinear element, Bell Sys. Tech. J. Part II 43 (1964) 1601-1608

[19] G. Szegö, R.E. Kalman, Sur la stabilité absolue d'un système d'équations aux différences finies, Comp. Rend. Acad. Sci. 257 no 2 (1963) 338-390

[20] E.I. Verriest, W. Aggoune, Stability of nonlinear differential delay systems, Mathematics and computers in Simulation 45 (1998) 257-267

[21] V.A. Yakubovich, Solution of certain matrix inequalities in the stability theory of nonlinear control systems, Dokl. Akad. Nauk. SSSR 143 (1962) 1304-1307 (English translation in Soviet Math. Dokl. 3 (1962) 620-623)

[22] G. Zames, On the input-output stability of nonlinear time-varying feedback systems, IEEE Trans. Automat. Contr. Part I 11 no 2 (1966) 228-238, Part II 11 no 3 (1966) 465-477

[23] K. Zhou, with J.C. Doyle, K. Glover, Robust and optimal control, Prentice Hall (1996) 


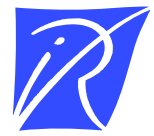

Unité de recherche INRIA Rocquencourt Domaine de Voluceau - Rocquencourt - BP 105 - 78153 Le Chesnay Cedex (France)

Unité de recherche INRIA Lorraine : LORIA, Technopôle de Nancy-Brabois - Campus scientifique 615, rue du Jardin Botanique - BP 101 - 54602 Villers-lès-Nancy Cedex (France)

Unité de recherche INRIA Rennes : IRISA, Campus universitaire de Beaulieu - 35042 Rennes Cedex (France)

Unité de recherche INRIA Rhône-Alpes : 655, avenue de l'Europe - 38330 Montbonnot-St-Martin (France)

Unité de recherche INRIA Sophia Antipolis : 2004, route des Lucioles - BP 93 - 06902 Sophia Antipolis Cedex (France)

INRIA - Domaine de Voluceau - Rocquencourt, BP 105 - 78153 Le Chesnay Cedex (France)

http://www.inria.fr

ISSN 0249-6399 